\title{
Succinylcholine: A Useful Drug in Bariatric Surgery
}

\author{
Jay B. Brodsky • Harry J. M. Lemmens • \\ John M. Morton
}

Received: 10 November 2008 / Accepted: 3 December 2008 /Published online: 17 December 2008

(C) Springer Science + Business Media, LLC 2008

To the editor:

We read the recent article by Tejirian et al. [1], and as members of our American College of Surgeons Level 1A Center of Excellence management team, we feel that a reply is indicated. Based on a single case, they recommended avoidance of succinylcholine (SCH) in all bariatric patients. At Stanford University, we take an opposite approach and use $\mathrm{SCH}$ to facilitate tracheal intubation for all of our morbidly obese patients.

With its rapid onset and short duration of action, $\mathrm{SCH}$ is ideal for rapid sequence induction of anesthesia. This is particularly important in bariatric patients because hemoglobin desaturation occurs rapidly following apnea and intubation of the trachea must be accomplished quickly [2]. SCH's onset is fast and its duration of action is short. Therefore, if difficulties

J. B. Brodsky $(\bowtie) \cdot$ H. J. M. Lemmens

Department of Anesthesia, Stanford University Medical Center, 300 Pasteur Drive,

Stanford, CA 94305, USA

e-mail: Jbrodsky@stanford.edu

J. M. Morton

Department of Surgery, Stanford University Medical Center, 300 Pasteur Drive,

Stanford, CA 94305, USA are encountered when securing the airway, the immediate return of motor function allows the patient to resume spontaneous ventilation.

There is no way to prove or disprove the "clinical" diagnosis that $\mathrm{SCH}$ was responsible for their patient's severe muscle aches. If $\mathrm{SCH}$ was the cause, it was an extreme presentation of a complication that is usually mild (if and when present) and of minimal clinical significance, especially in bariatric patients [3]. We have anesthetized hundreds of morbidly obese patients and thousands of other patients using $\mathrm{SCH}$ without ever having the serious complications they associated with its use.

As the authors' note, once the neuromuscular reversal agent sugammadex becomes available clinically, a nondepolarizing muscle relaxant such as rocuronium or vecuronium will become a safe option to $\mathrm{SCH}$. With sugammadex, reversal will be obtained immediately, effectively controlling time of paralysis. Until that time, we believe that $\mathrm{SCH}$ remains the drug of choice for tracheal intubation in bariatric patients.

\section{References}

1. Tejirian T, Lewis CE, Conner J, Jensen C, et al. Succinylcholine: a drug to avoid in bariatric surgery. Obes Surg. 2008; in press.

2. Brodsky JB, Lemmens HJM, Brock-Utne JG, et al. Morbid obesity and tracheal intubation. Anesth Analg 2002;94:732-6.

3. Lemmens HJM, Brodsky JB. The dose of succinylcholine in morbid obesity. Anesth Analg 2006;102:438-42. 